Pacific Journal of Mathematics

LINEAR MAPS ON SKEW SYMMETRIC MATRICES: THE
INVARIANCE OF ELEMENTARY SYMMETRIC FUNCTIONS 


\title{
LINEAR MAPS ON SKEW SYMMETRIC MATRICES: \\ THE INVARIANCE OF ELEMENTARY SYMMETRIC FUNCTIONS
}

\author{
Marvin Marcus and Roy Westwick
}

1. Introduction. Let $S_{n}$ be the space of $n$-square skew symmetric matrices over the field $F$ of real numbers. Let $E_{2 k}(A)$ denote the sum of all $2 k$-square principal subdeterminants of $A \in S_{n}$ (the elementary symmetric function of degree $2 k$ of the eigenvalues of $A$ ). It is classical that if $U$ is an $n$-square real orthogonal matrix and $A \in S_{n}$ then $U A U^{\prime} \in S_{n}$ and moreover for each $k$

$$
E_{2 k}\left(U A U^{\prime}\right)=E_{2 k}(A) .
$$

The correspondence

$$
A \rightarrow U A U^{\prime}
$$

for a fixed orthogonal $U$ can then be regarded as a linear transformation on $S_{n}$ onto itself that holds $E_{2 k}(A)$ invariant. The question we consider here is the following: to what extent does the fact that (1.1) holds for some $k$ characterize the map (1.2). In other words, we obtain (Theorem 3) the complete structure of those linear maps $T$ of $S_{n}$ into itself that for some $k>1$ satisfy $E_{2 k}(T(A))=E_{2 k}(A)$ for each $A \in S_{n}$. Our results are made to depend on the structure of linear maps of the second Grassmann product space $\Lambda^{2} U$ of a vector space $U$ over $F$ into itself.

K. Morita [2] examined the structure of those maps $T$ of $S_{n}$ into itself that hold invariant the dominant singular value $\alpha(A)$ of each $A \in S_{n}$. We recall that $\alpha(A)$ is the largest eigenvalue of the non-negative Hermitian square root of $A^{*} A$. Morita shows that if $\alpha(T(A))=\alpha(A)$ for each $A \in S_{n}$ then $T$ has essentially the form given in our Theorem 3 .

2. Some definitions and preliminary results. Let $U$ be a finite dimensional vector space of dimension $n$ over $F$. Let $G_{2}(U)$ denote the space of all alternating bilinear functionals on the cartesian product $U \times U$ to $F$. Then the dual space $\Lambda^{2} U$ of $G_{2}(U)$ is called the second Grassmann product space of $U$. If $x_{1}$ and $x_{2}$ are any two vectors in $U$ then $f=x_{1} \wedge x_{2} \in \wedge^{2} U$ is defined by the equation

$$
f(w)=w\left(x_{1}, x_{2}\right), \quad w \in G_{2}(U) .
$$

Received March 26, 1959. This research was supported by United States National Science Foundation Research Grant NSF G-5416. 
Some elementary properties of $x_{1} \wedge x_{2}$ are:

(i) $x_{1} \wedge x_{2}=0$ if and only if $x_{1}$ and $x_{2}$ are linearly dependent.

(ii) if $x_{1} \wedge x_{2}=y_{1} \wedge y_{2} \neq 0$ then $\left\langle x_{1}, x_{2}\right\rangle=\left\langle y_{1}, y_{2}\right\rangle$ where $\left\langle x_{1}, x_{2}\right\rangle$ is the space spanned by $x_{1}$ and $x_{2}$.

If $A$ is a linear map of $U$ into itself we define $C_{2}(A)$, the second compound of $A$, as a linear map of $\Lambda^{2} U$ into $\Lambda^{2} U$ by

$$
C_{2}(A) x_{1} \wedge x_{2}=A x_{1} \wedge A x_{2} .
$$

We remark that if $x_{1}, \cdots, x_{n}$ is a basis of $U$ then $x_{i} \wedge x_{j}, 1 \leq i<j \leq n$ is a basis of $\Lambda^{2} U$ and hence (2.1) defines $C_{2}(A)$ by linear extension. We first show that $\Lambda^{2} U$ is isomorphic in a natural way to $S_{n}$ and under this isomorphism second compounds correspond to congruence transformations in $S_{n}$.

Specifically, let $\alpha_{1}, \cdots, \alpha_{n}$ be a basis of $U$ and define $\varphi$ by

$$
\varphi\left(\alpha_{i} \wedge \alpha_{j}\right)=E_{i j}-E_{j i} \in S_{n}
$$

where $E_{i j}$ is the $n$-square matrix with 1 in position $i, j$ and 0 elsewhere and extend $\varphi$ linearly to all of $\Lambda^{2} U$. It is obvious that $\varphi$ is an isomorphism since $E_{i j}-E_{j i}, 1 \leq i<j \leq n$ is a basis of $S_{n}$. Let $T$ be a linear map of $\Lambda^{2} U$ into itself and define $S$, a linear map of $S_{n}$ into itself, by

$$
S(A)=\varnothing T \mathscr{P}^{-1}(A), A \in S_{n} .
$$

Let $B$ be a linear map of $U$ into itself. Then

THEOREM 1. $T=C_{2}(B)$ if and only if $S(A)=B_{1} A B_{1}^{\prime}$ where $B_{1}$ is the matrix of $B$ with respect to the ordered basis $\alpha_{1}, \cdots, \alpha_{n}$.

Proof. Suppose $T=C_{2}(B)$. Then for $i<j$

$$
\begin{aligned}
S\left(E_{i j}-E_{j i}\right) & =\varphi T \varphi^{-1}\left(E_{i j}-E_{j i}\right) \\
& =\varphi\left(B \alpha_{i} \wedge B \alpha_{j}\right) \\
& =\varphi\left(\sum_{k=1}^{n} b_{k i} \alpha_{k} \wedge \sum_{k=1}^{n} b_{k, j} \alpha_{k}\right) \\
& =\sum_{s, t} b_{s i} b_{t, j}\left(E_{s t}-E_{t s}\right) \\
& =B_{1}\left(E_{i j}-E_{j i}\right) B_{1}^{\prime} .
\end{aligned}
$$

The implication in the other direction is similar.

Let $L_{2 r}$ denote the set of rank $2 r$ matrices in $S_{n}$ and let $\Omega_{2 r}$ denote the set of vectors $\sum_{i=1}^{r} x_{i} \wedge y_{i}$ in $\Lambda^{2} U$ where $\operatorname{dim}\left\langle x_{1}, \cdots, x_{r}, y_{1}, \cdots, y_{r}\right\rangle=2 r$.

THEOREM 2. $\phi\left(\Omega_{2 r}\right)=L_{2 r}$ 
Proof. Let

$$
z=\sum_{i=1}^{r} x_{i} \wedge y_{i} \in \Omega_{2 r}
$$

Choose a non-singular map $B$ of $U$ onto $U$ such that $B \alpha_{2 j-1}=x_{j}$ and $B \alpha_{2 j}=y_{j}, j=1, \cdots, r$. Then

$$
z=C_{2}(B) \sum_{j=1}^{r} \alpha_{2 j-1} \wedge \alpha_{2 j}
$$

so

$$
\varphi(z)=\varphi C_{2}(B) \sum_{j=1}^{r} \alpha_{2 j-1} \wedge \alpha_{2 j} .
$$

Let $S(A)=B_{1} A B_{1}^{\prime}$ for $A \in S_{n}$ where $B_{1}$ is the matrix of $B$ with respect to the ordered basis $\alpha_{1}, \cdots, \alpha_{n}$. Then by Theorem $1, \varphi C_{2}(B) \varphi^{-1}=S$ and from (2.4) we have

$$
\begin{aligned}
\varphi(z) & =S \varphi \sum_{j=1}^{r} \alpha_{2 j-1} \wedge \alpha_{2 j} \\
& =S\left(\sum_{j=1}^{r}\left(E_{2 j-1,2 j}-E_{2 j, 2 j-1}\right)\right) \\
& =B_{1}\left(\sum_{j=1}^{r}\left(E_{2 j-1,2 j}-E_{2 j, 2 j-1}\right)\right) B_{1}^{\prime} \in L_{2 r} .
\end{aligned}
$$

The implication in the other direction is a reversal of this argument.

We see then that a map $T$ of $\Lambda^{2} U$ into itself is a second compound of some linear map of $U$ into itself if and only if $\varphi T \varphi^{-1}$ is a congruence map of $S_{n}$; and $T\left(\Omega_{2 r}\right) \subseteq \Omega_{2 r}$ if and only if $\varphi T \varphi^{-1}\left(L_{2 r}\right) \subseteq L_{2 r}$.

3. $E_{2 k}$ preservers. Let $S$ be a linear map of $S_{n}$ into itself such that $E_{2 k}(S(A))=E_{2 k}(A)$ for all $A \in S_{n}$, where $k$ is a fixed integer, $k \geq 2$. Then

Lemma $1 . \quad S$ is non-singular.

Proof. Suppose $S(A)=0$. Then

$$
\begin{aligned}
& E_{2 k}(A+X)=E_{2 k}(S(A+X))=E_{2 k}(S(X))=E_{2 k}(X) \\
& \text { for all } X \in S_{n} .
\end{aligned}
$$

Obtain a real orthogonal $P$ such that

$$
P A P^{\prime}=\sum_{i=1}^{r} \cdot\left(\begin{array}{lr}
0 & \theta_{i} \\
-\theta_{i} & 0
\end{array}\right)+0_{n-9 .}
$$

where $0_{n-2 r}$ is an $(n-2 r)$-square matrix of zeros and $\rho(A)=\operatorname{rank} A=2 r$. 
Here $\Sigma$ and $\dot{+}$ indicate direct sum. Now if $\rho(A) \geq 2 k$ simply set $X=0$ and from (3.1) and (3.2) we see that

$$
0<E_{2 k}(A)=E_{k}\left(\theta_{1}^{2}, \cdots, \theta_{r}^{2}\right)=E_{2 k}(0)=0
$$

a contradiction. On the other hand, if $\rho(A)<2 k$ select $X \in S_{n}$ such that

$$
P X P^{\prime}=0_{2 r}+\sum_{1}^{(k-r)}\left(E_{12}-E_{21}\right)+0_{n-2 k}
$$

where $E_{12}$ is a 2 -square matrix. Then

$$
E_{2 k}(A+X)=E_{2 k}\left(P A P^{\prime}+P X P^{\prime}\right)=\prod_{j=1}^{r} \theta_{j}^{2} .
$$

But $E_{2 k}\left(P X P^{\prime}\right)=E_{2 k}(X)=0$, since $k-r<k$. Hence the proof is complete.

Lemma 2. If $A \in S_{n}$ and $\operatorname{deg} E_{2 k}(x A+B) \leq 2$ for all $B \in S_{n}$ and $A \neq 0$ then $\rho(A)=2$.

Proof. Suppose $\rho(A)=2 r$ and select a real orthogonal $P$ such that $P A P^{\prime}$ has the form given in (3.2). Select $B$ such that

$$
P B P^{\prime}=0_{2 r}+\sum_{2}^{\left[\frac{n}{2}\right]-r} \cdot\left(E_{12}-E_{21}\right)+C
$$

where if $n$ is even $C$ doesn't appear and if $n$ is odd $C$ is a 1-square zero matrix.

Now if $k \leq r$

$$
\begin{aligned}
E_{2 k}(x A+B)= & x^{2 k} E_{k}\left(\theta_{1}^{2}, \cdots, \theta_{r}^{2}\right)+\text { lower } \\
& \text { order terms in } x .
\end{aligned}
$$

If $k>r$

$E_{2 k}(x A+B)=\left(\begin{array}{l}{[n / 2]-r} \\ k-r\end{array}\right) \theta_{1}^{2} \cdots \theta_{r}^{2} x^{2 r}+$ lower order terms in $x$. Thus

$$
\operatorname{deg} E_{2 k}(x A+B) \text { is either } 2 k \text { or } 2 r .
$$

But this implies $2 r=2$ and $\rho(A)=2$.

Lemma 3. If $E_{2 k}(S(A))=E_{2 k}(A)$ for all $A \in S_{n}$ then $S\left(L_{2}\right) \subseteq L_{2}$.

Proof. Let $p(x)$ be the polynomial $E_{2 k}(x A+B)$. Then if $\rho(A)=2$ it is easy to check that $\operatorname{deg} p(x) \leq 2$ for all $B \in S_{n}$. Hence $\operatorname{deg} E_{2 k}(x S(A)+S(B)) \leq$ 2 for all $B \in S_{n}$. But $S$ is non-singular by Lemma 1 and thus by Lemma 2 , $\rho(S(A))=2$. 
THEOREM 3. If $E_{2 k}(S(A))=E_{2 k}(A)$ for all $A \in S_{n}$, where $k$ is a fixed integer satisfying $4 \leq 2 k \leq n$ and $n \geq 5$ then there exists a real matrix $P$ such that

$$
S(A)=\alpha P A P^{\prime} \text { for all } A \in S_{n}
$$

where $\alpha P P^{\prime}=$ If $2 k<n$ and $\alpha P P^{\prime}$ is unimodular if $2 k=n$. If $2 k=$ $n=4$ then either $S$ has the form (3.3) or

$$
\begin{gathered}
S(A)=\alpha P\left(\begin{array}{cccc}
0 & a_{34} & a_{24} & a_{23} \\
-a_{34} & 0 & a_{14} & a_{13} \\
-a_{24} & -a_{14} & 0 & a_{12} \\
-a_{23} & -a_{13} & -a_{12} & 0
\end{array}\right) P^{\prime} \\
\text { where } A=\left(\begin{array}{cccc}
0 & a_{12} & a_{13} & a_{14} \\
-a_{12} & 0 & a_{23} & a_{24} \\
-a_{13} & -a_{23} & 0 & a_{34} \\
-a_{14} & -a_{24} & -a_{34} & 0
\end{array}\right) \text { and } \alpha P P^{\prime} \text { is unimodular. }
\end{gathered}
$$

Proof. By Lemma 1, $S^{-1}$ exists and we check that

$$
E_{2 k}\left(S^{-1}(A)\right)=E_{2 k}\left(S S^{-1}(A)\right)=E_{2 k}(A),
$$

for any $A \in S_{n}$. Hence by Lemma 3

$$
S^{-1}\left(L_{2}\right) \subseteq L_{2} \text { and thus } S\left(L_{2}\right)=L_{2} \text {. }
$$

Now define $T$, a mapping of $\Lambda^{2} U$ into itself, by (2.3)

$$
T=\varphi^{-1} S \varphi \text {. }
$$

By Theorem 2

$$
\begin{aligned}
T\left(\Omega_{2}\right) & =\varphi^{-1} S \varphi\left(\Omega_{2}\right) \\
& =\varphi^{-1} S\left(L_{2}\right) \\
& =\varphi^{-1}\left(L_{2}\right) \\
& =\Omega_{2} .
\end{aligned}
$$

At this point we invoke a theorem of Chow [1, pp. 38]. Let $T^{\prime \prime}$ be the mapping of 2-dimensional subspaces of $U$ into themselves induced by $T$; that is, let $T^{\prime \prime}(\langle x, y\rangle)=\langle u, v\rangle$ whenever $T(x \wedge y)=u \wedge v$, (assuming of course that $x$ and $y$ are linearly independent). Then $T^{\prime \prime}$ is well defined and it follows from the above that it is a one-to-one onto adjacence preserving transformation: if two 2-dimensional subspaces of $U$ intersect in a subspace of dimension 1 then their images under $T^{\prime \prime}$ intersect in a subspace of dimension 1 . Therefore $T^{\prime \prime}$ is induced either by a correlation or a collineation of the subspaces of $U$. If $\operatorname{dim} U \geq 5$ 
$T^{\prime \prime}$ is induced by a collineation. If $\operatorname{dim} U=4$ and if $T^{\prime \prime}$ is induced by a correlation then $\left(T T_{1}\right)^{\prime \prime}$ is induced by a collineation. Here $T_{1}$ maps $\Lambda^{2} U$ into itself and satisfies

$$
\begin{aligned}
& T_{1}\left(x_{i} \wedge x_{j}\right)=x_{l} \wedge x_{m}, \\
& \{i, j, l, m\}=\{1,2,3,4\} \text { and } i<j, l<m .
\end{aligned}
$$

Now, assuming $T^{\prime \prime}$ is induced by a collineation we show that

$$
T=\alpha C_{2}(P)
$$

for some $\alpha \in F$ and some linear transformation $P: U \rightarrow U$. The fundamental theorem of projective geometry states that there is a one-to-one semi-linear transformation $Q: U \rightarrow U$ such that

$$
T^{\prime \prime}(\langle x, y\rangle)=\langle Q x, Q y\rangle \text {. }
$$

Let $x_{1}, \cdots, x_{n}$ be a basis of $U$ and let $Q x_{i}=y_{i}$. Then

$$
\begin{aligned}
T\left(x_{i} \wedge x_{j}\right)=\alpha_{i j} y_{i} \wedge y_{j} \quad \alpha_{i j} \in F . \\
1 \leq i, \quad j \leq n, \quad i \neq j .
\end{aligned}
$$

Then for $s, k, t$ distinct integers in $1, \cdots, n$ and $K \in F$.

$$
\begin{aligned}
T\left(\left(x_{s}+x_{t}\right) \wedge x_{k}\right) & =K\left(Q\left(x_{s}+x_{t}\right) \wedge Q x_{k}\right) \\
& =K\left(y_{s}+y_{t}\right) \wedge y_{k},
\end{aligned}
$$

But

$$
\begin{aligned}
T\left(\left(x_{s}+x_{t}\right) \wedge x_{k}\right) & =T\left(x_{s} \wedge x_{k}\right)+T\left(x_{t} \wedge x_{k}\right) \\
& =\left(\alpha_{s k} y_{s}+\alpha_{t k} y_{t}\right) \wedge y_{k}
\end{aligned}
$$

Hence $\alpha_{s k}=\alpha_{t k}$ and thus $\alpha_{s k}=\alpha_{t k}=\alpha_{k t}=\alpha_{r t}=\alpha$ for any four distinct integers $s, k, r, t$. Hence

$$
T\left(x_{i} \wedge x_{j}\right)=\alpha y_{i} \wedge y_{j}=\alpha C_{2}(P) x_{i} \wedge x_{j},
$$

where $P: U \rightarrow U$ is a linear transformation with $P x_{j}=y_{i}$. Since $\left\{x_{i} \wedge x_{j} \mid 1 \leq\right.$ $i<j \leq n\}$ is a basis of $\Lambda^{2} U, T=\alpha C_{2}(P)$.

Now by Theorem 1,

$$
S(A)=\alpha P A P^{\prime} \text { for all } A \in S_{n}
$$

for $n \geq 5$ where $P$ is an $n$-square non-singular matrix. If $2 k=n$ then clearly $\alpha P P^{\prime}$ is unimodular. Hence assume $2 k<n$.

We next show that

$$
\alpha P P^{\prime}=I .
$$

From the hypothesis, 


$$
E_{2 k}\left(\alpha P A P^{\prime}\right)=E_{2 k}(A), A \in S_{n}
$$

and hence

$$
\alpha^{2 k} \operatorname{tr}\left\{C_{2 k}\left(P P^{\prime}\right) C_{2 k}(A)\right\}=\operatorname{tr} C_{2 k}(A) .
$$

By the polar factorization theorem let $P=U B$, where $U$ is real orthogonal and $B$ is positive definite symmetric. Let $B=V D V^{\prime}, D$ diagonal with positive entries and $V$ real orthogonal. Then since $V^{\prime} A V$ runs through all of $S_{n}$ as $A$ does we have

$$
\alpha^{2 k} \operatorname{tr}\left\{C_{2 k}\left(D^{2}\right) C_{2 k}(A)\right\}=\operatorname{tr} C_{2 k}(A) .
$$

We assert that any diagonal $\left(\begin{array}{l}n \\ 2 k\end{array}\right)$-square matrix is a linear combination of matrices $C_{2 k}(A)$ for $A \in S_{n}$. For, let $1 \leq i_{1},<\cdots<i_{2 k} \leq n$. Let $A \in S_{n}$ and consider the $2 k$-square principal submatrix $B$ of $A$ where

$$
B_{\alpha \beta}=A_{i_{\alpha i} i_{\beta}} ;
$$

and suppose $A$ has 0 entries outside of $B$. Then define $B$ as follows:

$$
\begin{array}{ll}
B_{2 k-\alpha, \alpha+1}=-1, & \alpha=0, \cdots, k-1 \\
B_{2 k-\alpha, \alpha+1}=1, & \alpha=k, \cdots, 2 k
\end{array}
$$

and $B_{i j}=0$ elsewhere. Then $C_{2 k}(A)= \pm E_{i_{1} \cdots i_{2 k}}$, where $E_{i_{1} \cdots i_{2 k}}$ is the $\left(\begin{array}{l}n \\ 2 k\end{array}\right)$-square matrix with the single non-zero entry 1 in the $\left(\left(i_{1}, \cdots, i_{2 k}\right)\right.$, $\left.\left(i_{1}, \cdots, i_{2 k}\right)\right)$ position ordered doubly lexicographically in the indices of the rows and columns of $A$. Returning to (3.9) we have

$$
\operatorname{tr}\left\{C_{2 k}\left(\alpha D^{2}\right) X\right\}=\operatorname{tr} X
$$

for all $\left(\begin{array}{l}n \\ 2 k\end{array}\right)$-square diagonal matrices $X$ and hence $C_{2 k}\left(\alpha D^{2}\right)=I, \alpha D^{2}=$ $\pm I$. From this we easily see that

$$
\alpha P P^{\prime}=I,
$$

and (3.3) follows. The mapping $T_{1}$ on $\Lambda^{2} U$ induces the map $S^{1}$ on $S_{4}$ where

$$
S^{1}\left(\begin{array}{cccc}
0 & a_{12} & a_{13} & a_{14} \\
-a_{12} & 0 & a_{23} & a_{24} \\
-a_{13} & -a_{23} & 0 & a_{34} \\
-a_{14} & -a_{24} & -a_{34} & 0
\end{array}\right)=\left(\begin{array}{cccc}
0 & a_{34} & a_{24} & a_{23} \\
-a_{34} & 0 & a_{14} & a_{13} \\
-a_{24} & -a_{12} & 0 & a_{12} \\
-a_{23} & -a_{13} & -a_{12} & 0
\end{array}\right)
$$

This completes the proof.

We remark that Theorem 3 is no longer valid if $k=1$ : for consider the transformation which interchanges positions $(i, j)$ and $(j, i)$ in $A$ for a fixed pair of integers $1 \leq i<j \leq n$. This clearly preserves $E_{2}(A)$ but 
does not have the form in Theorem 3. For example

$$
\left(\begin{array}{rrrr}
0 & 1 & 0 & 1 \\
-1 & 0 & 1 & 0 \\
0 & -1 & 0 & 1 \\
-1 & 0 & -1 & 0
\end{array}\right)
$$

is non-singular but interchanging the 1,2 and 2, 1 entries results in a singular matrix.

\section{REFERENCES}

1. Wei-Liang. Chow, On the Geometry of Algebraic Homogeneous Spaces, Annals of Math., 50 (1949), 32-67.

2. K. Morita, Schwarz's Lemma in a Homogeneous Space of Higher Dimensions, Japanese J. of Math. 19, (1944), 45-56.

The University of British Columbia, Vancouver, Canada 


\section{PACIFIC JOURNAL OF MATHEMATICS}

\section{EDITORS}

\author{
David Gilbarg \\ Stanford University \\ Stanford, California \\ F. H. Brownell \\ University of Washington \\ Seattle 5 , Washington
}

\author{
A. L. Whiteman \\ University of Southern California \\ Los Angeles 7, California \\ L. J. PAIGE \\ University of California \\ Los Angeles 24, California
}

\section{ASSOCIATE EDITORS}

\author{
E. F. BECKENBACH \\ T. M. CHERRY \\ D. DERRY
}
E. HEWITT
A. HORN
L. NACHBIN
M. OHTSUKA
H. L. ROYDEN
M. M. SCHIFFER

E. SPANIER

E. G. STRAUS

F. WOLF

\section{SUPPORTING INSTITUTIONS}

\author{
UNIVERSITY OF BRITISH COLUMBIA \\ CALIFORNIA INSTITUTE OF TECHNOLOGY \\ UNIVERSITY OF CALIFORNIA \\ MONTANA STATE UNIVERSITY \\ UNIVERSITY OF NEVADA \\ NEW MEXICO STATE UNIVERSITY \\ OREGON STATE COLLEGE \\ UNIVERSITY OF OREGON \\ OSAKA UNIVERSITY \\ UNIVERSITY OF SOUTHERN CALIFORNIA
}

\author{
STANFORD UNIVERSITY \\ UNIVERSITY OF TOKYO \\ UNIVERSITY OF UTAH \\ WASHINGTON STATE COLLEGE \\ UNIVERSITY OF WASHINGTON

AMERICAN MATHEMATICAL SOCIETY
CALIFORNIA RESEARCH CORPORATION
HUGHES AIRCRAFT COMPANY
SPACE TECHNOLOGY LABORATORIES
NAVAL ORDNANCE TEST STATION

Mathematical papers intended for publication in the Pacific Journal of Mathematics should be typewritten (double spaced), and the author should keep a complete copy. Manuscripts may be sent to any one of the four editors. All other communications to the editors should be addressed to the managing editor, L. J. Paige at the University of California, Los Angeles 24, California. ..

50 reprints per author of each article are furnished free of charge; additional copies may be obtained at cost in multiples of 50 .

The Pacific Journal of Mathematics is published quarterly, in March, June, September, and December. The price per volume (4 numbers) is $\$ 12.00$; single issues, $\$ 3.50$. Back numbers are available. Special price to individual faculty members of supporting institutions and to individual members of the American Mathematical Society: $\$ 4.00$ per volume; single issues, $\$ 1.25$.

Subscriptions, orders for back numbers, and changes of address should be sent to Pacific Journal of Mathematics, 2120 Oxford Street, Berkeley 4, California.

Printed at Kokusai Bunken Insatsusha (International Academic Printing Co., Ltd.), No. $6_{4}$ 2-chome, Fujimi-cho, Chiyoda-ku, Tokyo, Japan.

PUBLISHED BY PACIFIC JOURNAL OF MATHEMATICS, A NON-PROFIT CORPORATION

The Supporting Institutions listed above contribute to the cost of publication of this Journăl, but they are not owners or publishers and have no responsibility for its content or policies. 


\section{Pacific Journal of Mathematics}

\section{Vol. 10, No. $3 \quad$ November, 1960}

Glen Earl Baxter, An analytic problem whose solution follows from a simple

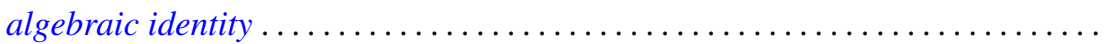

Leonard D. Berkovitz and Melvin Dresher, A multimove infinite game with linear payoff. .

Earl Robert Berkson, Sequel to a paper of A. E. Taylor ......................

Gerald Berman and Robert Jerome Silverman, Embedding of algebraic systems.... 767

Peter Crawley, Lattices whose congruences form a boolean algebra . . . . . ...... 777

Robert E. Edwards, Integral bases in inductive limit spaces . . . . . . . . . . . . . . .

Daniel T. Finkbeiner, II, Irreducible congruence relations on lattices . . . . . . . . . .

William James Firey, Isoperimetric ratios of Reuleaux polygons . . . . . . . . . . . 787

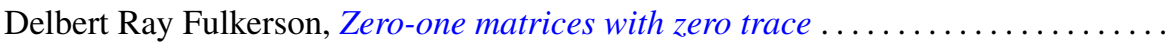

Leon W. Green, A sphere characterization related to Blaschke's conjecture........

Israel (Yitzchak) Nathan Herstein and Erwin Kleinfeld, Lie mappings in

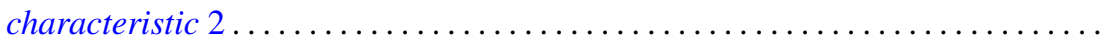

Charles Ray Hobby, A characteristic subgroup of a $p$-group .................

R. K. Juberg, On the Dirichlet problem for certain higher order parabolic

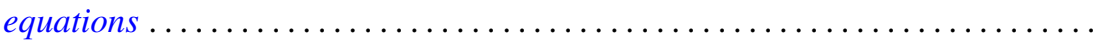

Melvin Katz, Infinitely repeatable games ......................

Emma Lehmer, On Jacobi functions . . . . . . . . . . . . . . . . . . . . . . . . .

D. H. Lehmer, Power character matrices

Henry B. Mann, A refinement of the fundamental theorem on the density of the sum of two sets of integers.

Marvin David Marcus and Roy Westwick, Linear maps on skew symmetric matrices: the invariance of elementary symmetric functions . .

Richard Dean Mayer and Richard Scott Pierce, Boolean algebras with ordered

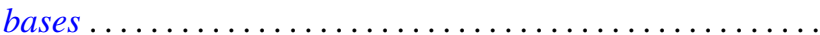

Trevor James McMinn, On the line segments of a convex surface in $E_{3} \ldots$

Frank Albert Raymond, The end point compactification of manifolds ..

Edgar Reich and S. E. Warschawski, On canonical conformal maps of regions of arbitrary connectivity

Marvin Rosenblum, The absolute continuity of Toeplitz's matrices...

Lee Albert Rubel, Maximal means and Tauberian theorems . .

Helmut Heinrich Schaefer, Some spectral properties of positive linear operators

Jeremiah Milton Stark, Minimum problems in the theory of pseudo-conformal transformations and their application to estimation of the curvature of the invariant metric.

Robert Steinberg, The simplicity of certain groups ...

Hisahiro Tamano, On paracompactness. .

Angus E. Taylor, Mittag-Leffler expansions and spectral theory .

Marion Franklin Tinsley, Permanents of cyclic matrices ...... . 